\title{
Search for Neutrinos from Gamma-Ray Bursts with the Baikal Neutrino Telescope NT200
}

A. V. Avrorin ${ }^{1}$, V. M. Aynutdinov ${ }^{1 *}$, I. A. Belolaptikov², D. Yu. Bogorodsky ${ }^{3}$, N. M. Budnev ${ }^{3}$, R. Wischnewski ${ }^{4}$, O. N. Gaponenko ${ }^{1}$, K. V. Golubkov ${ }^{2}$, O. A. Gress ${ }^{3}$, T. I. Gress ${ }^{3}$, O. G. Grishin ${ }^{3}$, I. A. Danilchenko', Zh.-A. M. Dzhilkibaev¹, G. V. Domogatsky ${ }^{1}$, A. A. Doroshenko1, A. N. Dyachok ${ }^{3}$, V. A. Zhukov', A. V. Zagorodnikov ${ }^{3}$, A. M. Klabukov ${ }^{1}$, A. I. Klimov ${ }^{5}$, K. V. Konishchev ${ }^{2}$, A. V. Korobchenko ${ }^{3}$,

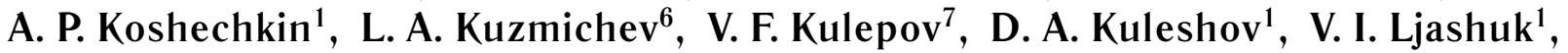
A. Middell ${ }^{4}$, M. B. Milenin ${ }^{7}$, R. R. Mirgazov ${ }^{1}$, S. P. Mikheev ${ }^{\dagger 1}$, E. A. Osipova ${ }^{6}$, A. I. Panfilov' ${ }^{1}$, L. V. Pan'kov ${ }^{3}$, G. L. Pan'kov' ${ }^{3}$ A. A. Perevalov ${ }^{3}$, D. A. Petukhov', E. N. Pliskovsky ${ }^{2}$, V. A. Poleshchuk ${ }^{1}$, E. G. Popova ${ }^{6}$, V. V. Prosin ${ }^{6}$, M. I. Rozanov ${ }^{8}$, V. Yu. Rubzov ${ }^{3}$, E. V. Rjabov ${ }^{3}$, O. V. Suvorova ${ }^{1}$, B. A. Tarashchansky ${ }^{3}$, S. V. Fialkovsky ${ }^{7}$, B. A. Shoibonov ${ }^{2}$, A. A. Sheifler ${ }^{2}$, A. V. Shirokov ${ }^{6}$, Ch. Spiering ${ }^{4}$ and A. S. Yagunov

${ }^{I}$ Institute for Nuclear Research, Russian Academy of Sciences, pr. 60-letiya Oktyabrya 7a, Moscow, 117312 Russia

${ }^{2}$ Joint Institute for Nuclear Research, ul. Joliot-Curie 6, Dubna, Moscow oblast, 141980 Russia

${ }^{3}$ Irkutsk State University, ul. Karla Marksa 1, Irkutsk, 664003 Russia

${ }^{4}$ Deutches Elektronen-Synchrotron, Platanenallee 6, D-15738 Zeuthen, Germany

${ }^{5}$ Russian Research Center Kurchatov Institute, pl. Kurchatova 1, Moscow, 123182 Russia

${ }^{6}$ Skobeltsyn Institute of Nuclear Physics, Moscow State University, Moscow, 119234 Russia

${ }^{7}$ Nizhny Novgorod State Technical University, ul. Minina 24, Nizhni Novgorod, 603950 Russia

${ }^{8}$ State Marine Technical University, Lotsmanskaya ul. 3, St.Petersburg, 190008 Russia Received February 24, 2011

\begin{abstract}
We present the results of our search for neutrino events coinciding in time and direction with gamma-ray bursts (GRBs) with the Baikal underwater neutrino telescope NT200. No events confirming a neutrino accompaniment of GRBs have been detected. Model-independent limits (Greens function) on the neutrino flux from GRBs have been obtained. For the Waxman-Bahcall neutrino spectrum, the limit on the neutrino flux from a GRB has been found to be $E_{\nu}^{2} \Phi_{\nu} \leqslant 1.1 \times 10^{-6} \mathrm{GeV} \mathrm{cm}^{-2} \mathrm{~s}^{-1} \mathrm{sr}^{-1}$.
\end{abstract}

DOI: $10.1134 / \mathrm{S} 106377371110001 \mathrm{X}$

Keywords: neutrino astrophysics, neutrino telescopes, gamma-ray bursts, Baikal.

\section{INTRODUCTION}

One of the priority goals of underwater neutrino telescopes related to the detection of neutrinos of extraterrestrial origin is the search for neutrino events from gamma-ray bursts (GRBs). In this paper, we present the results of such a search with the Baikal underwater neutrino telescope NT200. NT200 has

\footnotetext{
${ }^{\dagger}$ Deceased.

*E-mail: aynutdin@yandex.ru
}

been operating at the Baikal Lake since April 1998. The detector is located at a depth of $1100 \mathrm{~m}$. It consists of 192 optical modules (OMs) arranged on seven peripheral and one central string. The length of each string is $68 \mathrm{~m}$; the separation between the central and peripheral strings is $21.5 \mathrm{~m}$. To suppress the background triggers of the facility due to the dark current of the photodetectors and water luminescence, the optical modules of the telescope were grouped in pairs and switched in coincidence. Each such pair of modules forms a measuring channel. The 
time of the first triggered $\mathrm{OM}$ channel is chosen as the signal time stamp. A detailed description of the design and basic functional systems of the neutrino telescope NT200 is given in a number of publications of the Baikal collaboration (Belolaptikov et al. 1997; Aynutdinov et al. 2008a, 2008b).

To search for neutrino events, we used information about 303 GRBs recorded from 1998 to 2000 in the BATSE experiment (Paciesas et al. 1999). The technique for separating neutrinos from the background of atmospheric muons in the NT200 detector was based on the recording of muons from the Earth's lower hemisphere whose direction and recording time correlate with GRBs.

\section{EXPERIMENTAL DATA}

The information from the BATSE experiment about the GRB time and localization allows one to reduce considerably the background level of atmospheric muons in the neutrino telescope and to increase significantly the effective volume of the facility for the search of neutrino events. However, using the BATSE data is hampered by the fact that the information about the distance to the GRB source is available only for a small fraction of the events. The problem is that for GRB sources with a redshift $z \sim 2$ (the mean value that, according to present views, is expected for the BATSE events), a neutrino signal by a facility of the NT200 scale seems unlikely, i.e., only a relatively small fraction of the events from the BATSE data with $z$ considerably less than unity can be the source of the neutrino signal. An additional selection of GRBs by their duration (e.g., the selection of events with a duration shorter than $2 \mathrm{~s}$, for which the expected mean value of $z$ can be slightly lower than that for the complete BATSE data set) does not lead to a qualitative improvement of the situation either. In addition, the shortcoming of such a selection is the possibility of losing events with a statistically significant signal.

To prevent the loss of useful events, we used the complete BATSE data set from the catalog of triggered GRBs (Paciesas et al. 1999) without any selection of events by GRB parameters. The non-triggered GRBs extracted by an off-line analysis of the BATSE data (Stern and Tikhomirova, 2002) were added to these events. The absence of a trigger was associated either with the dead time of the detector or with an insufficient intensity of radiation.

For our analysis, we selected GRBs coinciding in time with periods of stable operation of the NT200 detector and located at zenith angles larger than $100^{\circ}$. The GRB selection results are given in Table 1. For each year of exposure, the total number of GRBs $\left(N_{\text {tot }}\right)$ recorded during the facility's stable operation
Table 1. Results of the GRB selection

\begin{tabular}{l|r|r|r|r|r|c}
\hline \multirow{2}{*}{ Parameter } & \multicolumn{2}{|c|}{ Triggered GRBs } & \multicolumn{3}{|c}{ Non-triggered GRBs } \\
\cline { 2 - 7 } & 1998 & 1999 & 2000 & 1998 & 1999 & 2000 \\
\hline$N_{\text {tot }}$ & 148 & 213 & 29 & 152 & 170 & 24 \\
$N_{100}$ & 60 & 84 & 11 & 60 & 78 & 10 \\
$\beta_{5}$ & 0.79 & 0.74 & 0.88 & 0.49 & 0.54 & 0.78 \\
$\beta_{10}$ & 0.94 & 0.92 & 0.98 & 0.77 & 0.80 & 0.95 \\
\hline
\end{tabular}

and the number of GRBs at zenith angles larger than $100^{\circ}\left(N_{100}\right)$ are presented. An important event parameter for the subsequent analysis is the error in the GRB direction given in the BATSE data. The data on the error allow one to determine the probability $\beta$ that the true GRB direction lies within the solid angle chosen for the search of neutrino events. For the selected GRBs, Table 1 lists the mean probabilities $\beta$ calculated for cones with half-angles of $5^{\circ}\left(\beta_{5}\right)$ and $10^{\circ}\left(\beta_{10}\right)$. To calculate these probabilities, we used an analytical representation of the GRB localization error distribution function from Briggs et al. (1999); its parameters are the directional error from the BATSE catalog and the systematic error taken to be $1.6^{\circ}$.

To search for correlations between GRBs and NT200 events, we used the data obtained with the Baikal neutrino telescope from 1998 (when NT200 was put into operation) to 2000 (when the BATSE experiment was completed). A primary analysis of the NT200 experimental information eliminated the periods of unstable operation of the facility and revealed a sample of events satisfying the $3 / 6$ trigger condition (no fewer than six triggered channels on three or more NT200 strings). From this sample, for the subsequent analysis we selected the events reconstructed as muons that arrived from below the horizon at zenith angles larger than $100^{\circ}\left(\sim 2.5 \times 10^{6}\right.$ events in $6.8 \times 10^{7} \mathrm{~s}$ of pure exposure time). The data set obtained includes mainly the background from muon bundles and misreconstructed muons near horizon. To suppress the background, we developed additional event selection criteria.

\section{NT200 EVENT SELECTION CRITERIA}

The optimization of the NT200 event selection criteria for the search of correlations with GRBs aimed achieving the maximum effective neutrino area, while retaining a sufficiently low background level from atmospheric muons. The optimization procedure was based on the simulations of neutrino events and of the background from atmospheric muons by the NT200 
Table 2. Fraction of the events satisfying the criterion $P_{\text {hit }} \times P_{\text {nohit }}$ for the muons from neutrinos and for background atmospheric muons

\begin{tabular}{|c|c|c|c|}
\hline Criterion & $P_{\text {hit }} \times P_{\text {nohit }}>0.1$ & $P_{\text {hit }} \times P_{\text {nohit }}>0.2$ & $P_{\text {hit }} \times P_{\text {nohit }}>0.3$ \\
\hline Atmospheric $\mu$ & 0.067 & 0.015 & 0.0043 \\
\hline Atmospheric $\nu$ & 0.80 & 0.69 & 0.52 \\
\hline$\nu: 10^{2} \mathrm{GeV}<E_{\nu}<10^{3} \mathrm{GeV}$ & 0.69 & 0.55 & 0.38 \\
\hline$\nu: 10^{3} \mathrm{GeV}<E_{\nu}<10^{4} \mathrm{GeV}$ & 0.66 & 0.51 & 0.33 \\
\hline$\nu: 10^{4} \mathrm{GeV}<E_{\nu}<10^{5} \mathrm{GeV}$ & 0.48 & 0.29 & 0.16 \\
\hline$\nu: 10^{5} \mathrm{GeV}<E_{\nu}<10^{6} \mathrm{GeV}$ & 0.23 & 0.12 & 0.062 \\
\hline$\nu: 10^{6} \mathrm{GeV}<E_{\nu}<10^{7} \mathrm{GeV}$ & 0.11 & 0.054 & 0.026 \\
\hline
\end{tabular}

facility (Belolaptikov 2007; Bugaev et al. 2001). To take into account the change in NT200 characteristics over the exposure period, the simulations were performed for nine basic configurations of the facility that corresponded most closely to the actual status of the detector recording system. The simulations produced the data samples of neutrino and background events for the NT200 detector satisfying the $3 / 6$ trigger condition.

We used the results of our reconstruction of simulated events to determine the efficiency of reconstructing the parameters of the trajectories for muons from neutrinos and to calculate the background level. The methods of event reconstruction and background suppression in NT200 were described in detail by Belolaptikov (2007). The criteria for suppressing the

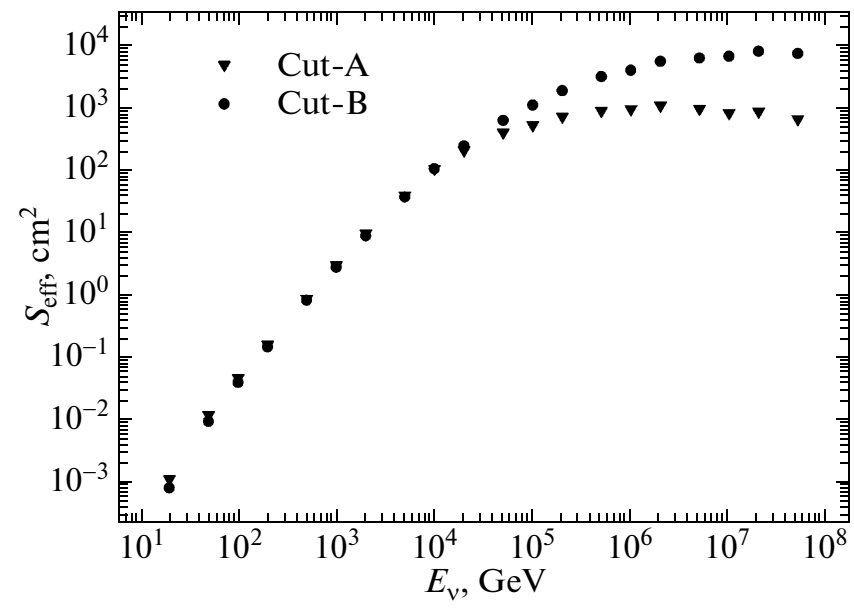

Fig. 1. Effective area of the NT200 facility versus neutrino energy for two sets of event selection criteria: A and B. background from atmospheric muons used here were developed and optimized for the separation of atmospheric neutrinos and provide a background suppression factor of $\sim 10^{-7}$. For the search of correlations with GRBs, this rejection level is redundant, because we have a priori information about the GRB recording time and direction. Reducing the level of requirements for the background suppression allows us to increase the probability of recording neutrino events and to increase considerably the effective area of the facility. Following the approaches by Belolaptikov (2007), we chose $P_{\text {hit }} \times P_{\text {nohit }}$ and $Z_{\text {dist }}$ as the basic parameters for the selection of events. The quantity $Z_{\text {dist }}$ is calculated as the distance between the most distant projections of the coordinates of the facility's triggered channels onto the muon trajectory. $P_{\text {hit }} \times$ $P_{\text {nohit }}$ is the normalized probability that the triggered channels of the facility for the chosen particle trajectory would record the signal from the Cherenkov radiation of a muon, while the nontriggered ones would not record the signal. The largest admissible angle $\Psi$ between the GRB direction and the reconstructed direction of muon motion is also an optimization parameter.

A decrease of the angular window $\Psi$ obviously leads to a reduction in the admixture of background events. However, when $\Psi$ decreases considerably, the fraction of the losses of useful events becomes significant and exceeds $50 \%$ for $\Psi<5^{\circ}$. Table 2 illustrates the application of the criterion $P_{\text {hit }} \times P_{\text {nohit }}$. This criterion was developed to suppress the background from atmospheric muons misreconstructed as events from below the horizon and to separate atmospheric neutrinos at energies up to $\sim 1 \mathrm{TeV}$. As the neutrino energy increases, the efficiency of this criterion decreases. The data from Table 2 characterize the level of losses of useful events for atmospheric neutrinos 
and for neutrinos with a spectrum of the form $E^{-2}$ in several energy ranges. The fraction of losses at high neutrino energies can be more than $90 \%$.

Table 3 presents the number of background events over the exposure time calculated for various values of $P_{\text {hit }} \times P_{\text {nohit }}$ and $\Psi$.

Based on our simulations, we chose two sets of selection criteria for NT200 events for their subsequent comparison with GRBs, Cut-A and Cut-B:

Cut-A: $Z_{\text {disk }}>30 \mathrm{~m}, P_{\text {hit }} \times P_{\text {nohit }}>0.1, \Psi=10^{\circ}$;

Cut-B: $Z_{\text {disk }}>30 \mathrm{~m}, \Psi=5^{\circ}$.

Cut-A is tailored to search for neutrinos with energies up to $\sim 10^{5} \mathrm{GeV}$ : the number of expected background events is close to unity. Cut-B, which does not use the criterion $P_{\text {hit }} \times P_{\text {nohit }}$, extends considerably the energy range for the search of neutrino events, but the expected background level increases approximately by a factor of 4 .

\section{THE EFFECTIVE AREA OF THE FACILITY}

The effective area of the facility for the Cut-A and Cut-B event selection criteria is presented in Fig. 1. The effective areas for the two sets of selection criteria are identical up to neutrino energies of $\sim 10^{4} \mathrm{GeV}$. At energies above $10^{5} \mathrm{GeV}$, the effective area for Cut-A does not increase with energy, because the fraction of the losses of useful events rises. The behavior of the effective area at energies above $10^{6} \mathrm{GeV}$ is mainly determined by the absorption of neutrinos in the Earth.

The range of energies to which the NT200 detector is sensitive was estimated for a neutrino spectrum of the form $E^{-2}$. Figure 2 shows the relation between the NT200 effective area and the neutrino energy. For the Cut-A and Cut-B criteria, the energy range is limited from above by $\sim 10^{6}$ and $\sim 10^{7} \mathrm{GeV}$, respectively.

\section{RESULTS OF OUR ANALYSIS OF THE EXPERIMENTAL DATA}

We analyzed the experimental data along three main directions: checking the correctness of the facility response simulation procedure and the calculation of the detector effective area; separating the events correlating with GRBs in time and direction; and determining the number of background events. To check the simulation procedure, the calculated background event suppression factors were compared with the experimentally obtained values. Table 4 presents the results for various values of $P_{\text {hit }} \times P_{\text {nohit }}\left(Z_{\text {dist }}=\right.$ $30 \mathrm{~m})$. The experimental background suppression factors obtained are consistent with the simulation results within the systematic errors of our calculations, $\sim 20 \%$.
Table 3. Expected number of background events

\begin{tabular}{c|c|c|c}
\hline Parameter & $\begin{array}{c}P_{\text {hit }} \times P_{\text {nohit }} \\
\geqslant 0\end{array}$ & $\begin{array}{c}P_{\text {hit }} \times P_{\text {nohit }} \\
>0.1\end{array}$ & $\begin{array}{c}P_{\text {hit }} \times P_{\text {nohit }} \\
>0.2\end{array}$ \\
\hline$\Psi=5^{\circ}$ & 3.1 & 0.20 & 0.047 \\
$\Psi=10^{\circ}$ & 12 & 0.80 & 0.18 \\
\hline
\end{tabular}

Table 4. Experimental estimate of the background event suppression factor

\begin{tabular}{l|c|c|c}
\hline Parameter & $\begin{array}{c}P_{\text {hit }} \times P_{\text {nohit }} \\
>0.1\end{array}$ & $\begin{array}{c}P_{\text {hit }} \times P_{\text {nohit }} \\
>0.2\end{array}$ & $\begin{array}{c}P_{\text {hit }} \times P_{\text {nohit }} \\
>0.3\end{array}$ \\
\hline Experiment & 0.053 & 0.012 & 0.0035 \\
Model & 0.062 & 0.014 & 0.0040 \\
\hline
\end{tabular}

We searched for correlations of the NT200 events selected according to the Cut-A and Cut-B criteria for 303 GRBs ( 155 triggered and 148 non-triggered GRBs). The selection by the event recording time was made within the time interval $T_{\mathrm{GRB}}$ determined by the value of $T_{90}$, the GRB duration presented in the GRB catalogs. To compensate for the possible uncertainty in the recording time of an NT200 event, we added $5 \mathrm{~s}$ to the interval $T_{90}$ on each side. If there was no information about $T_{90}$ (about $25 \%$ of the triggered GRBs), we used a fixed time interval of $100 \mathrm{~s}$.

The number of background events was determined in a time interval of $\pm 1000 \mathrm{~s}$ relative to the GRB onset (the interval $T_{\mathrm{GRB}}$ was excluded in this case) for angles between the GRB and the muon $<10^{\circ}$. The number of background events obtained was normalized to the recording time and the angular range defined for the signal.

Our analysis revealed no event for Cut-A and one event for Cut-B (GRB 990427 from the BATSE catalog). Table 5 gives the number of events for the signal and background matching the selection criteria; the $90 \%$ confidence intervals for $\mu_{90}$ obtained in accordance with Feldman and Cousins (1998); the number of GRBs with the correction $\beta$ applied (the probability that the true GRB position is within the chosen solid angle, see Table 1 ); the $90 \%$ confidence intervals for the number of events per GRB: $N_{90}=$ $\mu_{90} /\left(N_{\mathrm{GRB}} \times \beta\right)$. The results are presented both for all GRBs and separately for the triggered GRBs.

Our analysis revealed no neutrino events from GRBs. We obtained the limit on the neutrino flux from GRBs based on the method proposed by Fukuda et al. (2002). According to this technique, the $F\left(E_{v}\right)$ 


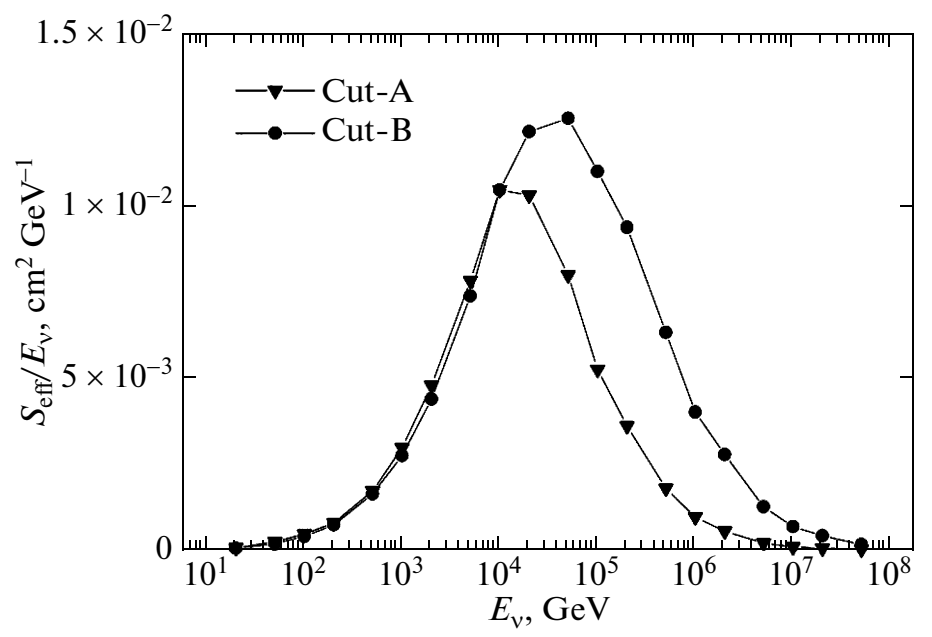

Fig. 2. Ratio of the effective area of neutrino recording by the NT200 detector to the neutrino energy.

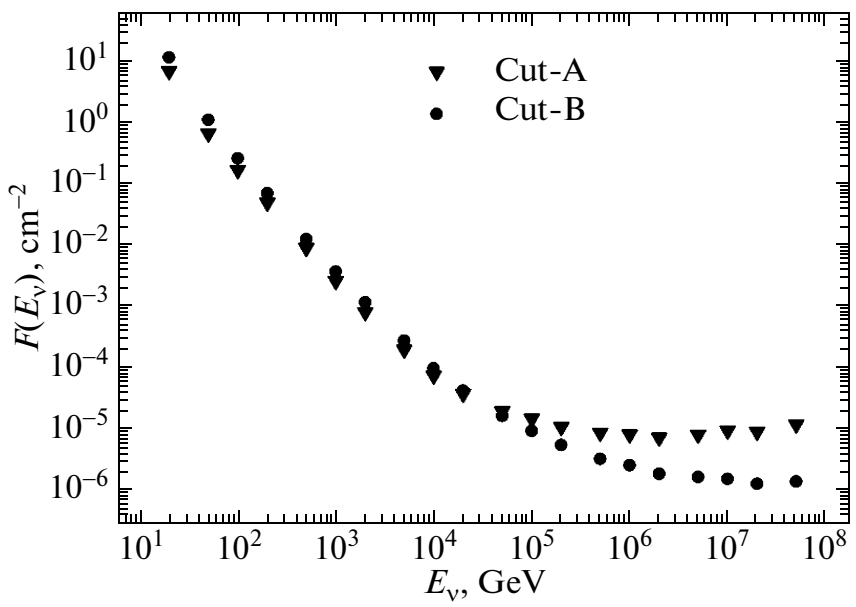

Fig. 3. Limits on the neutrino flux from GRBs (the Greens function) obtained for two event selection methods, Cut-A and Cut-B.

limit is represented as a function of neutrino energy (the Greens function):

$$
F\left(E_{\nu}\right)=N_{90} / S_{\text {eff }}\left(E_{\nu}\right),
$$

where $S_{\text {eff }}\left(E_{\nu}\right)$ is the effective area of the facility, $N_{90}$ is the $90 \%$ confidence interval for the number of neutrino events per GRB. In this approach, the result does not depend on the assumptions about the form of the neutrino energy spectrum. Figure 3 presents the $F\left(E_{\nu}\right)$ limits for the Cut-A and Cut-B selection criteria obtained in our analysis. The limits for Cut-A and Cut-B essentially coincide at energies up to $10^{5} \mathrm{GeV}$. At high neutrino energies, the Cut-B selection criteria yield considerably better results.

In Fig. 4, the results for the NT200 detector are compared with the limits on the neutrino flux from GRBs obtained with Super-Kamiokande (Fukuda et al. 2002) and AMANDA (Achterberg et al. 2007a, 2007b). The limit for the AMANDA detector was obtained for GRBs in the Northern Hemisphere. The Super-Kamiokande and NT200 limits refer to GRBs in the Southern Hemisphere.

At present, there is no unique form of the energy spectrum for neutrinos from GRBs. Therefore, the Greens function $F\left(E_{\nu}\right)$, the dependences of the neutrino flux limit on neutrino energy, are our modelindependent experimental result. The Greens function allow us to calculate the limit on the neutrino flux for energy spectra of an arbitrary form. We performed such a calculation for the neutrino spectrum from Waxman and Bahcall (1997) and Waxman (2001, 2007). Following the approaches from these papers, the differential muon neutrino flux $\Phi_{\nu}^{W-B}\left(E_{\nu}\right)$ at energies up to $10 \mathrm{PeV}$ is

$$
E_{\nu}^{2} \Phi_{\nu}^{W-B}\left(E_{\nu}\right)=A^{W-B} \times \min \left(1, E_{\nu} / E_{\nu b}\right),
$$


Table 5. Results of searching for events from GRBs

\begin{tabular}{l|c|c|c|c|c}
\hline \multicolumn{1}{c|}{ Selection criteria } & Experiment & Background & $\mu_{90}$ & $N_{\mathrm{GRB}} \times \beta$ & $N_{90}$ \\
\hline Cut-A & 0 & 0.56 & 1.9 & 236 & 0.0085 \\
Cut-B & 1 & 2.7 & 2.1 & 199 & 0.010 \\
Cut-B, triggered GRBs & 1 & 1.6 & 2.8 & 120 & 0.023 \\
\hline
\end{tabular}

where $E_{\nu b}=100 \mathrm{TeV}$ and $A^{W-B}=8 \times$ $10^{-9} \mathrm{GeV} \mathrm{cm}^{-2} \mathrm{~s}^{-1} \mathrm{sr}^{-1}$.

The MRF (Model Rejection Factor) was calculated from the formula

$$
\mathrm{MRF}=N_{90} / N_{\mathrm{ex}},
$$

where $N_{90}$ is the upper limit on the number of neutrino events from a GRB and $N_{\text {ex }}$ is the expected number of events calculated for a given spectrum:

$$
N_{\mathrm{ex}}=\int \Phi_{\nu}^{\text {Earth }}\left(E_{\nu}\right) S_{\text {eff }}\left(E_{\nu}\right) \frac{4 \pi}{n} d E_{\nu}
$$

In this formula, $n \approx 2.2 \times 10^{-5} \mathrm{~s}^{-1}$ is the mean rate of GRB observation in a solid angle of $4 \pi$ ( $\sim 700$ events per year in the BATSE recording range) and $\Phi_{\nu}^{\text {Earth }}\left(E_{\nu}\right)$ is the neutrino flux near the Earth: $\frac{\Phi_{\nu}^{W-B}\left(E_{\nu}\right)}{2}$.

Since the expected number of events was estimated from information about the rate of GRB observations corresponding to the BATSE recording range, the $M R F$ was calculated only for triggered GRBs $\left(N_{\mathrm{GRB}} \times \beta=120\right.$, see Table 5$)$. The MRF obtained is $2.8 \times 10^{2}$ and the limit on the neutrino flux from GRBs is

$$
E_{\nu}^{2} \Phi_{\nu} \leq 1.1 \times 10^{-6} \mathrm{GeV} \mathrm{cm}^{-2} \mathrm{~s}^{-1} \mathrm{sr}^{-1} .
$$

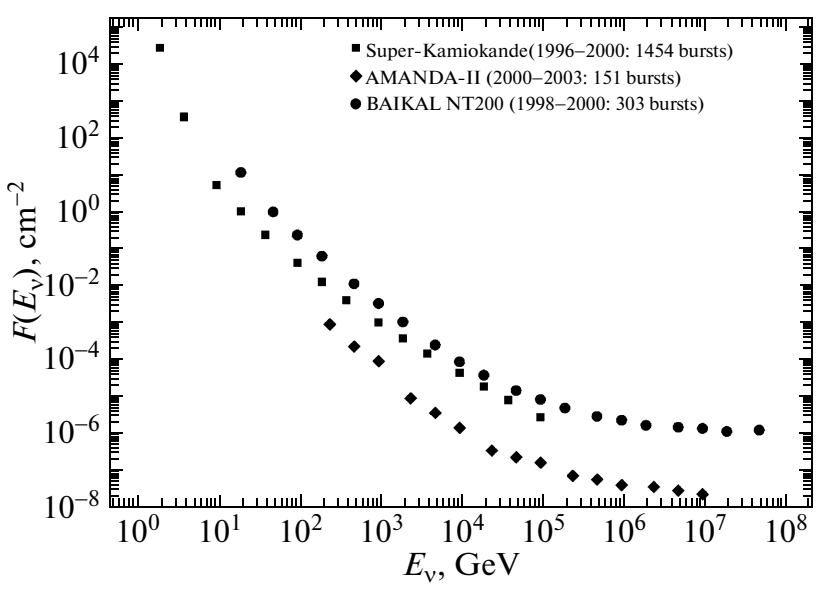

Fig. 4. Limits on the neutrino flux from GRBs obtained on NT200, Super-Kamiokande, and AMANDA.
The derived limit on the neutrino flux is considerably weaker than the AMANDA and IceCube results obtained for GRBs in the Northern Hemisphere: by now, IceCube has reached a level of $M R F<1$ (Abbasi et al. 2011). An improvement of the experimental situation is possible once the neutrino telescopes with an effective volume of the order of a cubic kilometer in the Mediterranean Sea and at Lake Baikal have been put into operation (Avrorin et al. 2011), which together with IceCube record neutrinos from GRBs on the full celestial sphere.

\section{CONCLUSIONS}

We presented the results of our search for neutrino events in the underwater neutrino telescope NT200 coinciding in time and direction with GRBs recorded by the BATSE detector from 1998 to 2000. The angular range of the NT200 sensitivity to GRBs spans a large part of the Southern Hemisphere. No events confirming the neutrino accompaniment of GRBs were detected. Model-independent limits on the neutrino flux from GRBs (the Greens function) were obtained in an energy range that exceeds the SuperKamiokande range by more than an order of magnitude. For the neutrino energy spectrum proposed by Waxman and Bahcall (1997), the limit on the neutrino flux from GRBs was found to be $E_{\nu}^{2} \Phi_{\nu} \leqslant$ $1.1 \times 10^{-6} \mathrm{GeV} \mathrm{cm}^{-2} \mathrm{~s}^{-1} \mathrm{sr}^{-1}$.

\section{ACKNOWLEDGMENTS}

This work was supported by the Ministry of Science and Education of the Russian Federation (GK 02.740.11.0018, GK 02.518.11.7158, P1242, P2504, P133, P878, P1146, RNP 2.2.1.1/1483, RNP 2.1.1/1539, RNP 2.2.1.1/5901, REK “Baikal”), the Ministry of Science and Education of Germany, and the Russian Foundation for Basic Research (project nos. 08-02-00432, 08-02-00198, 09-0210001, 09-02-00623, 09-02-12295, 10-02-10007). 


\section{REFERENCES}

1. R. Abbasi, Y. Abdou, T. Abu-Zayyad, et al., Phys. Rev. Lett. 106141101 (2011); astro-ph/1101.1448.

2. A. Achterberg, M. Ackermann, J. Adams, et al., Astrophys. J. 664, 397 (2007a).

3. A. Achterberg, M. Ackermann, J. Adams, et al., astro-ph/0705.1186 (2007b).

4. A. Avrorin, V. Aynutdinov, and I. Belolaptikov, Nucl. Instrum. Methods Phys. Res. Sec. A 639, 30 (2011).

5. V. Aynutdinov, A. Avrorin, V. Balkanov, et al., Astropart. Phys. 29, 366 (2008a).

6. V. Aynutdinov, A. Avrorin, V. Balkanov, et al., Nucl. Instrum. Methods Phys. Res. Sec. A 588, 99 (2008b).

7. I. A. Belolaptikov, Preprint IYaI RAN 1178 (2007).

8. I. A. Belolaptikov, L. B. Bezrukov, B. A. Borisovets, et al., Astropart. Phys. 7, 263 (1997).

9. M. S. Briggs, G. N. Pendleton, R. M. Kippen, et al., astro-ph/9901111 (1999).
10. E. V. Bugaev, S. I. Klimushin, and I. A. Sokalski, Phys. Rev. D 64, 4016 (2001).

11. G. Feldman and R. Cousins, Phys. Rev. D 57, 3873 (1998).

12. S. Fukuda, Y. Fukuda, M. Ishitsuka, et al., Astrophys. J. 578, 317 (2002).

13. W. S. Paciesas, C. A. Meegan, G. N. Pendleton, et al., Astrophys. J. Suppl. Ser. 122465 (1999).

14. B. Stern and Ya. Tikhomirova, www.astro.su.se/groups/head/grb archive.html (2002).

15. E. Waxman, astro-ph/0103186 (2001).

16. E. Waxman, Phil. Trans. R. Soc. London A 3651323 (2007).

17. E. Waxman and J. Bahcall, Phys. Rev. Lett. 782292 (1997).

Translated by G. Rudnitskii 